\title{
Assessment of level of physical activity and cardiovascular responses to submaximal treadmill exercise test inhealthy post graduate students.
}

\author{
Hongprachan Hungyo, ${ }^{1}$ Sarada N, ${ }^{2}$ Ak Brogen Singh, ${ }^{3}$ Leivon Bellamy \\ Kom, ${ }^{1}$ Ashem Nandarani Devi, ${ }^{1}$ Kanmi Ningshen, ${ }^{1}$ Sharon Roel. \\ ${ }^{1}$ Merrycka A. sangma. \\ ${ }^{1}$ Post graduate students, Department of Physiology, RIMS ${ }^{2 .}$ Associate Professor, Department of Physiology, \\ RIMS. ${ }^{3 .}$ Professor, Department of Community Medicine. Regional Institute of Medical Sciences Imphal, \\ Manipur, India.
}

\begin{abstract}
Sedentary lifestyle and physical inactivity is a world-wide health problem increasing the morbidity and mortality of the population in developing countries. The aim of the study was to assess and evaluate the level of physical activity and cardiovascular responses following aerobic exercise.Method: The study was focussed to a group of post-graduate students and House officers working in the tertiary referral hospital. 165 (female $=30 ;$ male $=135$ ) with mean age (male $=31.40 \pm 5.73$; female $=30.2 \pm 5.63$ ) were subjected to Bruce Modified Treadmill Test (BMTT) along with Questionnaires (Short term last 7 day Recall Self-administered format. Statistical analysis:All values are given as means $\pm S D$. Analysis of statistical significance for variablesmainly heart rate (HR), blood pressure (BP) (pre, during and post TMT test), and METs values were done by one-way analysis of variance (ANOVA). Paired ' $t$ ' test and independent ' $t$ ' test for different variables for

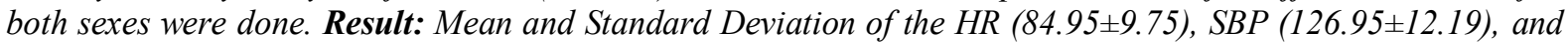

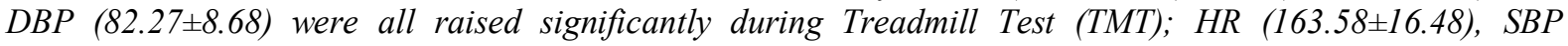
(152.71 \pm 11.76$)$ and $D B P(85.69 \pm 9.66)$ and unable to return to normal even after 5 minutes recovery period, whereas the DBP returned to lower than normal after 3 minutes recovery period (81.98 \pm 8.26$)$. Significant correlation of BMI with all other variables i.e.HR, SBP, and DBP (pre, during and post TMT) were noted except a negative correlation with TMT-HR (Significance $=0.447$ ) was noted. There was significant correlation

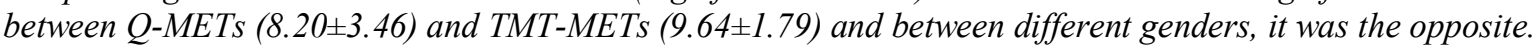

Key words: Physical activity, METs, TMT, HR, SBP, DBP.

\section{Introduction:}

Physical activity is defined as any bodily movement produced by skeletal muscles that results in energy expenditure. ${ }^{1}$ It is also defined for non-pregnant, non-lactating adults as that person's total energy expenditure in a 24 hour period divided by his or her basal metabolic rate (BMR). ${ }^{1}$ Energy expenditure is expressed in terms of calories. One calorie is defined as the quantity of heat necessary to raise the temperature of $1 \mathrm{~kg}(1 \mathrm{~L})$ of water. Physical inactivity is responsible for a large world-wide burden of the disease and health care costs. ${ }^{2}$ There is direct relationship between physical activity and risk reduction for non-communicable diseases. The magnitude of these diseases continues to rise, especially in low and middle income countries. As the age increases, the intensity level of physical activity decreases. ${ }^{3}$

The autonomic nervous system plays a vital role in the regulation of the cardiovascular system. HR recovery after Treadmill exercise test is an indicator of parasympathetic activity. ${ }^{4}$ Cardiac output, the total blood volume pumped by the left ventricle of the heart per minute is the product of heart rate and stroke volume and so during exercise there is dramatic changes in coronary circulation, heart rate and blood pressure.Konstantin Dipla ${ }^{5}$ observed that an exaggerated blood pressure response during acute dynamic exercise i.e.increase in systolic blood pressure from rest $>10 \mathrm{mmHg}$ had been considered as an indicator of cardiovascular risk.

There is no gold standard for physical activity level measurement. Subjective methods, such as Questionnaires, interviews etc. are usually preferred in epidemiological studies. ${ }^{6}$ These methods are faster and less tedious to fill in but affected by recall bias. Level of physical activity can be assessed by knowing the term MET (Metabolic Equivalent Task). 1 MET is considered as resting metabolic rate obtained during quiet sitting. ${ }^{7}$ Physical activity is associated with age, body mass, health and other lifestyle factors.Dynamic exercise is often used to evaluate the functions of cardiovascular system and the Treadmill Test is a commonly used dynamic exercise protocol. 


\section{Materials \& Methods:}

It is a cross sectional study carried out among post graduate students and House officers working in various departments of a tertiary referral hospital, Imphal, India. 165 subjects in the age group 26 to 48 years participated in the 7 day recall Questionnaires followed by sub-maximal Modified Bruce Protocol Treadmill Test using Vega 201 Stress Test System, Recorders and Medicare System (p) Ltd. Chandigarh; approval and clearance from ethics committee being sought. Methods and procedure of the test was explained and demonstrated to the participants before conducting test. Room temperature was maintained between $18-27^{\circ} \mathrm{C}$. The subjects were allowed to take complete rest in supine position for about 10 minutes and then all the basal/resting measurable variables were recorded. Exercise was terminated on achieving $85 \%$ of the age and sex predicted maximal heart rate (HR) or achieving more than 7.0 METs value or depending on the subjects own discretion. The parameters/variables studied were body mass index (BMI), HR, systolic blood pressure (SBP), diastolic blood pressure (DBP) and energy expenditure in terms of METs value and estimated METs value from the questionnaires. Three readings - pre exercise, during exercise and post exercise (Recovery) after 3 minutes for variables HR, SBP, and DBP were recorded.

\section{III.Results:}

As seen from table 1 and 2 there was significant increase in both HR, SBP and DBP in all phases but slight decrease in recovery phase for DBP. In the study all the variables are higher in males $(n=135)$ as compared to females $(\mathrm{n}=30)$.

There was significant positive correlation of BMI with all other variables except a negative correlation with HR during TMT test METs value. Statistically there was significant correlation between METs values derived from Questionnaires and TMT test(table 3 and 4).All the participants could attain vigorous level of physical activity i.e. (> 6 METs) but their physical activity is not correlated with BMI. Rather there was negative correlation (Significance $=0.907)$.

Table I. Mean variables according to age and sex.

\begin{tabular}{|c|c|c|c|c|c|}
\hline No. of Subjects & $\begin{array}{c}\text { Age (years) } \\
\mathrm{x} \pm \mathrm{SD}\end{array}$ & $\begin{array}{c}\text { Height }(\mathrm{cm}) \\
\mathrm{x} \pm \mathrm{SD}\end{array}$ & $\begin{array}{c}\text { Weight }(\mathrm{kg}) \\
\mathrm{x} \pm \mathrm{SD}\end{array}$ & $\begin{array}{c}\text { BMI } \\
\mathrm{x} \pm \mathrm{SD}\end{array}$ & $\begin{array}{c}\text { METs } \\
\mathrm{x} \pm \mathrm{SD}\end{array}$ \\
\hline Male (135) & $31.40 \pm 5.73$ & $167.17 \pm 6.50$ & $70.21 \pm 9.39$ & $25.08 \pm 2.37$ & $9.74 \pm 1.79$ \\
\hline Female (30) & $30.2 \pm 5.63$ & $154.8 \pm 3.43$ & $57.73 \pm 6.3$ & $24.25 \pm 2.24$ & $8.98 \pm 1.78$ \\
\hline
\end{tabular}

Table II. Mean Treadmill Test values; Mean \pm SD (Range); *Significant; **Highly Significant $*(\mathrm{p}<0.05) ; * *(\mathrm{p}<0.01)$

\begin{tabular}{|l|l|l|l|}
\hline Variables & Pre-test & During test & Post-test \\
\hline HR & $84.95 \pm 9.75$ & $163.58 \pm 16.48^{* *}$ & $116.34 \pm 12.76^{* *}$ \\
& $(83.45-86.45)$ & $(161.05-166.12)$ & $(114.38-118.30)$ \\
\hline SBP & $126.95 \pm 12.19$ & $152.71 \pm 11.76^{* *}$ & $136.35 \pm 13.88^{* *}$ \\
& $(125.07-128.28)$ & $(150.90-154.52)$ & $(134.21-138.48)$ \\
\hline DBP & $82.27 \pm 8.68$ & $85.69 \pm 9.66^{* *}$ & $81.98 \pm 8.26^{* *}$ \\
& $(80.93-83.60)$ & $(84.21-87.18)$ & $(80.70-83.25)$ \\
\hline
\end{tabular}

Table III. Correlation of BMI with HR, SBP, DBP and TMT-METs; (Total no. of participants $=165$ )

$*$ (Significance $\mathrm{p}<0.05) ; * *$ (Significance $\mathrm{p}<0.01$ )

\begin{tabular}{|l|l|l|}
\hline Variables & r- value & Significance \\
\hline Pre-test HR & $0.316^{* *}$ & 0.000 \\
\hline TMT-HR & -0.060 & 0.447 \\
\hline Recovery HR & 0.068 & 0.387 \\
\hline Pre-test SBP & $0.410^{* *}$ & 0.000 \\
\hline TMT-SBP & $0.265^{* *}$ & 0.001 \\
\hline Recovery SBP & $0.235^{* *}$ & 0.002 \\
\hline Pre-test DBP & $0.337^{* *}$ & 0.000 \\
\hline TMT-DBP & $0.327^{* *}$ & 0.000 \\
\hline Recovery DBP & $0.283^{* *}$ & 0.000 \\
\hline
\end{tabular}


Table IV. Correlation between Q-METs and TMT-METs

\begin{tabular}{|l|l|l|l|l|}
\hline & Mean \pm SD & $\begin{array}{l}\text { r- } \\
\text { value }\end{array}$ & t-value & Significance \\
\cline { 1 - 2 } Q-MET & $8.20 \pm 3.46$ & 0.169 & -5.114 & 0.000 \\
\hline TMT-MET & $9.64 \pm 1.79$ & & & \\
\hline
\end{tabular}

\section{Discussion:}

Physical activity is associated with body mass and other factors but not correlated with BMI. ${ }^{8} \mathrm{HR}$ response during dynamic exercise is a powerful and independent predictor of adverse cardiovascular events during long-term follow-up among healthy men and women.A very rapid HR recovery immediately after exercise was associated with lower risk of CHD and CVD events. ${ }^{4}$ Attenuated heart rate response during exercise is associated with adverse cardiovascular outcome. ${ }^{9}$

Diastolic blood pressure returned to normal or slightly lower after 3 minutes during recovery period whereas HR and SBP were still on the higher side even after 5 minutes of rest following exercise test. Raised blood pressure was observed in some participants whose age ranged between (30-50) years whose BMI were also correspondingly increased. During exercise HR and SBP were raised whereas DBP remained same or fell or increased slightly from mean. However, there was exaggerated DBP responsein those who had more BMI and higher resting DBP as compared to other participants. These findings were similar to the studies of Pande S $\mathrm{S}$ et al. ${ }^{10}$

In another group of participants whose initial DBP raised upto $100 \mathrm{mmHg}$ but normal BMI showed substantial decreased in DBP during exercise-the reason may be decreased peripheral resistance leading to fall in DBP due to peripheral vasodilation. In the long term, there is compensatory splanchnic sympathetic vasoconstriction maintaining constant diastolic blood pressure. Another explanation is that themean arterial pressure increases linearly with increasing rates of HR and work load in response to treadmill exercise. This increased mean arterial pressure is mainly due to systolic pressure as diastolic pressure remains at near-resting levels. Since mean arterial pressure is equivalent to total peripheral resistance and cardiac output, the increased in cardiac output outweighs the concomitant decreased in peripheral resistance. Questionnaires and TMT are reliable subjective and objective means for assessing physical activity level. Biased responses are the setbacks of 7-day recall Self-administered Questionnaires.

\section{Conclusion:}

Meeting physical activity guidelines decline with age. Variables of age, sex and BMI are the most influencing factors with more males meeting physical activity guidelines than females. However there is not much significant result with BMI. ${ }^{11}$ The finding, according to the present study, suggested that most of the doctors in the study group attained vigorous intensity level of physical activity. It was also found that the level of physical activities assessed from the Questionnaires, were well correlated with the findings from TMT. Physical activity is associated with body mass but not correlated with it. Questionnaires and Treadmill Test are reliable means for assessing level of physical activity in spite of some limitations such as biased responses, well equipped setting required for TMT etc.

\section{Acknowledgements:}

Hereby, we would like to appreciate friendsfor their helpful suggestions. We arealso very much grateful to those who participated in the study and help us complete this study.

\section{References:}

[1]. Caspersen CJ, Powell KE, Christenson GM et al. Physical Activity, Exercise and Physical Fitness: Definitions and distinctions for health related research. Public Health Resp Mar-Apr 1985; 100(2): 126-31.

[2]. Barbosa N, Sanchez CE, Vera JA, Perez W, Thalabard JC and Rieu M. A physical activity questionnaire: Reproducibility and Validity. Journal of Sports Science and Medicine 2007; 6: 505-18.

[3]. Global Status Report on Non-communicable Diseases WHO Jan-March 2012; 1-123.

[4]. Morshedi-Meibodi A, Larson M G, Levy D, O’Donnell C J, and Vasan R S. Heart Rate Recovery After Treadmill Exercise Testing and Risk of Cardiovascular Disease Events ( The Framingham Heart Study). Am J Cardiol October 2002; 90:848-852.

[5]. Dipla K, Nassis G P, and Vrabas I S. Blood Pressure Control at Rest and during Exercise in Obese Children and Adults. Journal of Obesity 2012; ID 147385: 10 .

[6]. Kriska A Caspersen C. Introduction to a Collection of Physical Activity Questionnaires. Med Sci Sports Exerc 1997; $29(6)$ : S5 -S9. 
[7]. Ainsworth B E, William L et al. Compendium of Physical Activities: an update of activity codes and MET intensities. Med Sci Sports Exerc. 2000;s 32( 9): S498-S516

[8]. Norman A Bellocco R, Vaida F Wolk A. Total physical activity in relation to age, body mass, health and other factors in a cohort of Sweden men. Int. J Obs 2002; 26:670-5.

[9]. Maor E, Kopel E, Sidi Y, Goldenberg I, Segev S,and Kivity S. Effect of Mildly Attenuated Heart Rate Response During Treadmill Exercise Testing on Cardiovascular Outcome in Healthy Men and Women. Am J Cardiol 2013; x: x-x.

[10]. Pande SS, Pande SR,Dhore RB, Dhaphale AV, Parate VR, Patel SS et al. Assessment of cardiovascular Responses to Treadmill Exercise in Normal Healthy Indian Adolescents. Indian J Physiol Pharmacol 2012; 56(1):36-41.

[11]. Vasickova J, Fromel K, Nykodyn J. Physical activity recommendation and its Association with Demographic Variables in Czech University Students. Acta Univ. Palacki. Olomuc, Gymn 2008; 38(2). 